\title{
Review of Experimental Concepts for Studying the Quantum Vacuum Field
}

\author{
E. W. Davis ${ }^{1}$, V. L. Teofilo ${ }^{2}$, B. Haisch ${ }^{3}$, H. E. Puthoff ${ }^{1}$, L. J. Nickisch ${ }^{4}$, A. \\ Rueda $^{5}$, and D. C. Cole \\ ${ }^{1}$ Inst. for Advanced Studies at Austin, 4030 W. Braker Ln., Ste. 300, Austin, TX 78759, USA \\ ${ }^{2}$ Lockheed Martin Space Systems, M.S. L2-01/B157, PO Box 3504, Sunnyvale, CA 94089, USA \\ ${ }^{3}$ ManyOne Networks, 100 Enterprise Way, Bldg. G-370, Scotts Valley, CA 95066, USA \\ ${ }^{4}$ NorthWest Research Associates, 14508 NE $20^{\text {th }}$ St., Bellevue, WA 98007, USA \\ ${ }^{5}$ Dept. of Electrical Engineering, ECS Bldg., Cal. State Univ.-Long Beach, Long Beach, CA 90840, USA \\ ${ }^{6}$ Dept. of Manufacturing Engineering, Boston University, 15 St. Mary's Street, Boston, MA 02215, USA \\ 1512-342-2187,ewdavis@earthtech.org
}

\begin{abstract}
We review concepts that provide an experimental framework for exploring the possibility and limitations of accessing energy from the space vacuum environment. Quantum electrodynamics (QED) and stochastic electrodynamics (SED) are the theoretical approaches guiding this experimental investigation. This investigation explores the question of whether the quantum vacuum field contains useful energy that can be exploited for applications under the action of a catalyst, or cavity structure, so that energy conservation is not violated. This is similar to the same technical problem at about the same level of technology as that faced by early nuclear energy pioneers who searched for, and successfully discovered, the unique material structure that caused the release of nuclear energy via the neutron chain reaction.
\end{abstract}

Keywords: Zero-point Fluctuations, Quantum Vacuum, Quantum Electrodynamics, Stochastic Electrodynamics PACS: 03.50.De, 05.10.Gg, 12.20.-m

\section{INTRODUCTION}

Quantum theory predicts that the vacuum of space in the universe is filled with low-energy electromagnetic waves, random in phase and amplitude and propagating in all possible directions. This is different from the cosmic microwave background radiation and it is referred to as the electromagnetic quantum vacuum since it is the lowest state of otherwise empty space. When integrated over all frequency modes up to the Planck frequency, $v_{\mathrm{P}}\left(\sim 10^{43}\right.$ $\mathrm{Hz}$ ), this represents an enormous potential source of energy with a density of as much as $\sim 10^{113} \mathrm{~J} / \mathrm{m}^{3}$ which is far in excess of any other known energy source even if only an infinitesimal fraction of it is accessible. This is also several tens of orders of magnitude greater than the energy density of matter-antimatter annihilation reactions. Even if we are constrained to integrate over all frequency modes only up to the nucleon Compton frequency $\left(\sim 10^{23} \mathrm{~Hz}\right)$, this energy density will still be enormous $\left(\sim 10^{35} \mathrm{~J} / \mathrm{m}^{3}\right)$. And we have not taken into account the fact that the electromagnetic quantum vacuum is not alone by itself. On the contrary, it intimately couples to the charged particles in the Dirac sea of particle-antiparticle pairs and thereby couples to the other interactions of the Standard Model (weak and strong force vacua). So all the numbers we just mentioned admit of some further adjustment.

This energy is so enormous that most physicists believe that even though zero-point energy (ZPE) seems to be an inescapable consequence of quantum field theory, it cannot be physically real, and so is subtracted away in calculations by ad hoc means. A minority of physicists do, however, accept it as a real energy which we cannot directly sense since it is the same everywhere, even inside our bodies and measuring devices. From this perspective, the ordinary world of matter and energy is like foam atop the quantum vacuum sea. It does not matter to a ship how 
deep the ocean is below it. If the ZPE is real, then there is the possibility that it can be tapped as a source of power or be harnessed to generate a propulsive force for space travel.

The propeller or the jet engine of an aircraft can push air backwards to propel the aircraft forward. A ship or boat propeller does the same thing in water. On Earth there is always air or water available to push against. Ignoring mass fluctuations or other propellant concepts, a rocket in space has nothing to push against, and so it needs to carry propellant to eject in the absence of air or water. The fundamental problem is that a deep space rocket would have to start out with all the propellant it will ever need. This quickly results in the need to carry more and more propellant just to propel the propellant. The breakthrough one wishes for in deep space travel is to overcome the need to carry propellant at all. How can one generate a propulsive force without carrying and ejecting propellant?

There is a force associated with the electromagnetic quantum vacuum: the Casimir force (Casimir, 1948). This force is an attraction between parallel uncharged metallic plates that has now been well measured (Lamoreaux, 1997; Mohideen, 1998; Chen et al., 2004) and can be attributed to a minute imbalance in the zero-point energy density inside the cavity between the plates versus the region outside the plates as shown in Figure 1. However, this is not useful for propulsion since it symmetrically pulls on the plates. If some asymmetric variation of the Casimir force could be identified, though, then one could in effect sail through space as if propelled by a kind of quantum fluctuation wind. Unfortunately, at this point this is pure speculation since it requires an invention to contrive such a means.

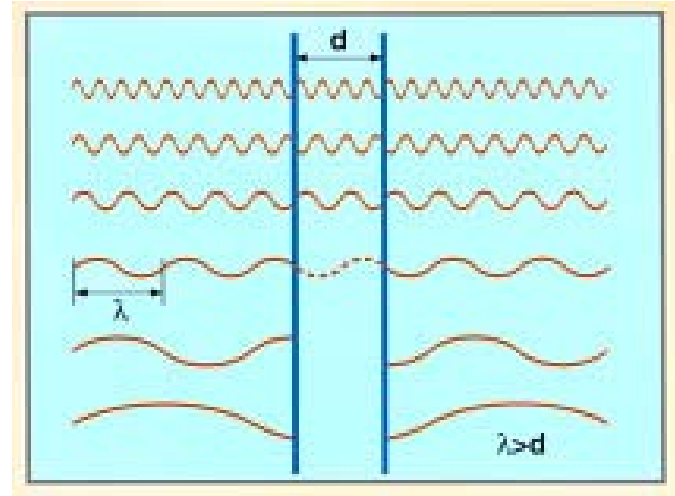

FIGURE 1. Casimir Effect ( $\mathrm{d}=$ cavity wall separation, $\lambda=$ ZPF mode wavelength).

The other requirement for space travel is energy. It is sometimes assumed that attempting to extract energy from the vacuum zero-point field (ZPF) would somehow violate the laws of thermodynamics. Fortunately, it turns out that this is not the case. A thought experiment published by Forward $(1983 ; 1984)$ demonstrated how the Casimir force could in principle be used to extract energy from the vacuum ZPF. He showed that any pair of conducting plates at close distance experiences an attractive Casimir force that is due to the electromagnetic ZPF of the vacuum. A "vacuum-fluctuation battery" can be constructed by using the Casimir force to do work on a stack of charged conducting plates (see Figure 2). By applying a charge of the same polarity to each conducting plate, a repulsive electrostatic force will be produced that opposes the Casimir force. If the applied electrostatic force is adjusted to be always slightly less than the Casimir force, the plates will move toward each other and the Casimir force will add energy to the electric field between the plates. The battery can be recharged by making the electrical force slightly stronger than the Casimir force to re-expand the foliated conductor.

Cole and Puthoff (1993) verified that (generic) energy extraction schemes are not contradictory to the laws of thermodynamics. For thermodynamically reversible processes, no heat will flow at temperature $\mathrm{T}=0$. However, for thermodynamically irreversible processes, heat can be produced and made to flow, either at $\mathrm{T}=0$ or at any other $\mathrm{T}>0$ situation, such as by taking a system out of mechanical equilibrium. Moreover, work can be done by or done on physical systems, either at $\mathrm{T}=0$ or $\mathrm{T}>0$ situations, whether for a reversible or irreversible process. However, if one is considering a net cyclical process on the basis of, say, the Casimir Effect, then energy would not be able to be continually extracted without a violation of the second law of thermodynamics. Thus, Forward's process cannot be 
cycled to yield a continuous extraction of energy. Here, the recharging of the battery would, owing to frictional and other losses, require more energy than is gained from the ZPF. There is no useful engine cycle in this process; nonetheless, the plate-contraction phase of the cycle does demonstrate the ability to cause "extraction" of energy from the ZPF. It does reflect work done by the ZPF on matter.

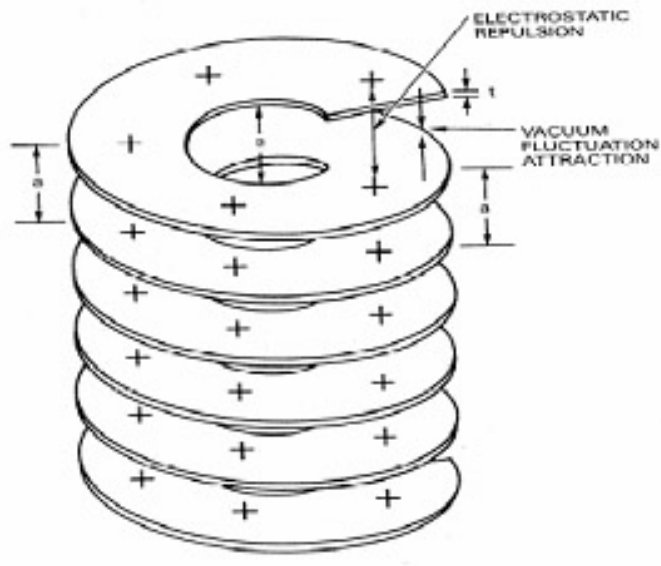

FIGURE 2. Vacuum-Fluctuation Battery (Forward, 1983, 1984).

Another illustrative example of a scheme for extracting energy from the ZPF is a patent by Mead and Nachamkin (1996). They propose that a set of resonant dielectric spheres be used to extract energy from the ZPF and convert it into electrical power. They consider the use of resonant dielectric spheres, slightly detuned from each other, to provide a beat-frequency downshift of the more energetic high-frequency components of the ZPF to a more easily captured form. Their device includes a pair of dielectric structures that are positioned proximal to each other and which intercept incident ZPE radiation. The volumetric sizes of the structures are selected so that they resonate at a particular frequency of the incident radiation. But the volumetric sizes of the structures are chosen to be slightly different so that the secondary radiation emitted from them at resonance interferes with each other, thus producing a beat frequency radiation that is at a much lower frequency than that of the incident radiation, and that can be converted into electrical energy. A conventional metallic antenna (loop or dipole type, or a RF cavity structure) can then be used to collect the beat frequency radiation. This radiation is next transmitted from the antenna to a converter via an electrical conductor or waveguide and converted to electrical energy. The converter must include: 1) a tuning circuit or comparable device so that it can effectively receive the beat frequency radiation, 2) a transformer to convert the energy to electrical current having a desired voltage, and 3) a rectifier to convert the energy to electrical current having a desired waveform. See Figure 3.

The receiving structures are composed of dielectric material in order to diffract and scatter the incident ZPE radiation. The volumetric sizing requirements for the receiving structures are selected to enable them to resonate at a high frequency of the incident ZPE radiation based on the parameters of frequency (of the incident ZPE radiation) and propagation characteristics of the medium (vacuum or otherwise) and of the receiving structures. Since the ZPE radiation energy density increases with increasing frequency, greater amounts of electromagnetic energy are potentially available at higher frequencies. Consequently, the size of the receiving structures must be miniaturized in order to produce greater amounts of energy from a system located within a space or area of a given size. Therefore, the smaller the size of the receiving structures, the greater the amount of energy that can be produced by the system. No experimental study has been performed to validate this invention and characterize its performance, or otherwise confirm or refute its claimed efficacy.

Although novel ZPF energy extraction mechanisms have been proposed in the literature (some credible, many not credible), no practicable technique has been successfully demonstrated in the laboratory. Therefore, our mission is to work with the theoretical physicists who developed the comprehensive understanding of ZPF theory along with 
experienced electrodynamic and RF engineers so that we can further characterize the physics of the ZPF and identify possible energy extraction techniques and test their feasibility for application to space power systems. In particular, we are pursuing experimental designs that are capable of either confirming or refuting the theoretical predictions and expectations by many experimentalists that cyclic energy can be obtained from the ZPF. Our proposed research program is to: 1) develop a theoretical framework for analyzing and developing the potential ZPF energy and extend original research as needed; 2) design and assess potential methods and techniques utilizing ZPF energy as a practical power source; 3) prepare detailed test plans for candidate experiments; 4) design and fabricate ZPF energy extraction device(s) for performance characterization and validation; 5) test the devices under appropriate space conditions, as necessary; 6) evaluate test results for application to space power and propulsion; and 7) identify necessary additional development for qualification of ZPF energy devices. In what follows, we summarize the physics of the ZPF and the experimental investigation we propose to conduct in support of our program.
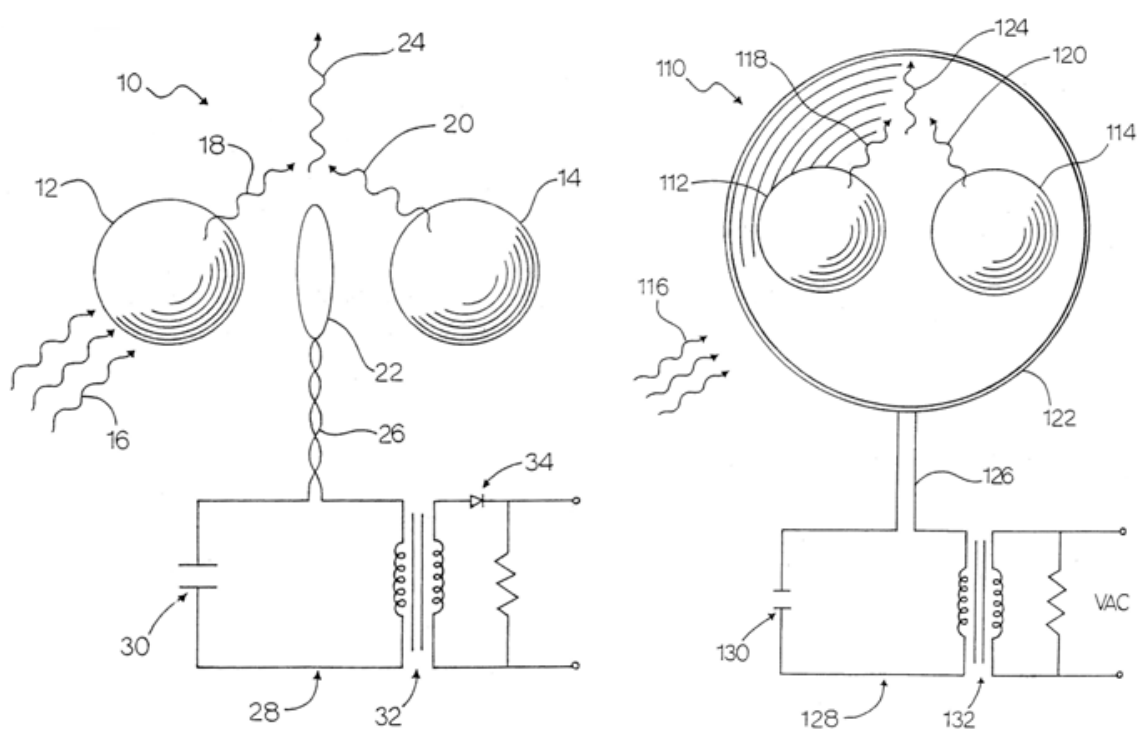

FIGURE 3. ZPE Resonant Dielectric Spheres Electrical Power Generator (Mead and Nachamkin, 1996).

The schematic at left shows one embodiment of the invention that uses a metallic dipole antenna (item 22) and conductor (item 26) to transmit the beat frequency radiation energy to the electrical power converter (items $28,30,32,34$ ). Item 16 is the incident

ZPE radiation; items 12 and 14 are the dielectric receiving structures; items 18, 20 and 24 are the secondary emitted radiation.

The schematic at right shows a second embodiment of the invention that uses a RF cavity (item 122) and waveguide (item 126) to transmit the beat frequency radiation energy to the electrical power converter (items 128, 130,132). Item 116 is the incident ZPE radiation; items 112 and 114 are the dielectric receiving structures; items 118,120 and 124 are the secondary emitted radiation.

\section{ORIGIN OF ZERO-POINT FIELD ENERGY}

In the traditional quantum theory presented in many textbooks, the basis of the ZPF is attributed to the so-called Heisenberg Uncertainty Principle. According to this principle, A and B are any two conjugate observables that we are interested in measuring in a lab experiment and they must obey the commutation relation $[\mathrm{A}, \mathrm{B}]=\mathrm{i} \hbar \mathrm{h}$ is Planck's reduced constant). Their corresponding uncertainty relation is $\Delta \mathrm{A} \Delta \mathrm{B} \geq \hbar / 2$, where $\Delta \mathrm{A}$ is the variance (a.k.a. uncertainty) of observable $\mathrm{A}$ and $\Delta \mathrm{B}$ is that of the conjugate observable $\mathrm{B}$. This relation states that if one measures observable $\mathrm{A}$ with very high precision (i.e., its uncertainty $\Delta \mathrm{A}$ is very small), then a simultaneous measurement of observable $\mathrm{B}$ will be less precise (i.e., its uncertainty $\Delta \mathrm{B}$ is very large), and vice versa. In other words, it is not possible to simultaneously measure two conjugate observable quantities with infinite precision. This minimum uncertainty is not due to any correctable flaws in measurement, but rather reflects the intrinsic fuzziness in the quantum nature of energy and matter. Substantial theoretical and experimental work has shown that in many quantum systems the limits to measurement precision is imposed by the quantum vacuum zero-point fluctuations (ZPF) embodied within the uncertainty principle. Nowadays we rather see the Heisenberg Uncertainty Principle as a necessary consequence, and therefore, a derived result of the wave nature of quantum phenomena. The uncertainties 
are just a consequence of the Fourier nature of conjugate pairs of quantities (observables). For example, the two Fourier-wave-conjugates time and frequency become the pair of quantum-particle conjugates time and energy and the two Fourier-wave-conjugates displacement and wavenumber become the pair of quantum-particle conjugates position and momentum. For more on this see, e.g., Peres (1993).

Radio and microwaves, infrared light, visible light, ultraviolet light, X-rays, and gamma rays are all forms of electromagnetic radiation. Classically, electromagnetic radiation can be pictured as waves flowing through space at the speed of light. The waves are not waves of anything substantive, but are in fact ripples in the state of a field. These waves carry energy, and each wave has a specific direction, frequency and polarization state. This is called a "propagating mode of the electromagnetic field." A useful tool for modeling the (propagating mode of the) electromagnetic field in quantum mechanics is the ideal quantum mechanical harmonic oscillator: a hypothetical mass on a perfect spring oscillating back and forth under the action of the spring's restoring force, which is small enough to be subject to quantum laws. The Heisenberg Uncertainty Principle dictates that a quantized harmonic oscillator (a.k.a. a photon state) can never come entirely to rest, since that would be a state of exactly zero energy, which is forbidden. Every mode of the field must have $\hbar \omega / 2(\omega$ is the mode/photon frequency, $\hbar \omega$ is the energy of a single mode/photon) as its average minimum energy in the vacuum. (This is a tiny amount of energy, but the number of modes is enormous, and indeed increases as the square of the frequency. The product of this tiny energy per mode times the huge spatial density of modes yields a very high theoretical energy density per unit volume.) It is for this reason that a ZPE term is added to the classical blackbody spectral radiation energy density $\rho(\omega) \mathrm{d} \omega$ (i.e., the energy per unit volume of radiation in the frequency interval $[\omega, \omega+d \omega])$ for when the absolute temperature $T$ of the oscillator system becomes $0 \mathrm{~K}$ in the vacuum (Milonni, 1994):

$$
\rho(\omega) d \omega=\frac{\omega^{2}}{\pi^{2} c^{3}}\left[\frac{\hbar \omega}{e^{\hbar \omega / k T}-1}+\frac{\hbar \omega}{2}\right] d \omega
$$

where $\mathrm{c}$ is the speed of light, and $\mathrm{k}$ is Boltzmann's constant (note: $\omega=2 \pi v$ ). The factor outside the square brackets is the density of (mode/photon) states (i.e., the number of states per unit frequency interval), the first term inside the square brackets is the standard Planck blackbody radiation energy per mode, and the second term inside the square brackets is the quantum zero-point energy per mode. Equation (1) is called the Zero-Point Planck (ZPP) spectral radiation energy density.

From this line of reasoning, quantum physics predicts that all of space must be filled with electromagnetic zeropoint fluctuations (a.k.a. the zero-point field) creating a universal sea of zero-point energy. The density of this energy depends critically on where the frequency of the zero-point fluctuations cease. Since space itself is thought to break up into a kind of quantum foam at a tiny distance scale called the Planck length, $1_{\mathrm{P}}\left(\sim 10^{-35} \mathrm{~m}\right)$, it is argued that the zero-point fluctuations must cease at the corresponding $v_{\mathrm{P}}$. If that is the case, then the zero-point energy density would be 108 orders of magnitude greater than the radiant energy at the center of the Sun. That is the extreme limit. Formally, in QED the ZPE energy density is taken as infinite. However, arguments based on quantum gravity considerations yield a finite cutoff at $\nu_{\mathrm{p}}$. Therefore, the spectral energy density is given by $\rho(\omega) \mathrm{d} \omega$ $=\left(\hbar \omega^{3} / 2 \pi^{2} c^{3}\right) \mathrm{d} \omega$, which integrates to an energy density, $\mathrm{u}=\hbar v_{\mathrm{p}}^{4} / 8 \pi^{2} \mathrm{c}^{3} \approx 10^{113} \mathrm{~J} / \mathrm{m}^{3}$. As large as the ZPE is, interactions with it are typically cut off at lower frequencies depending on the particle coupling constants or their structure.

In SED the origin of the ZPF comes as a direct consequence of the fundamental assumptions. SED is just the ordinary classical electrodynamics of Maxwell and Lorentz where instead of taking the homogeneous solution of the source-free differential wave equations for the electromagnetic potentials, as done in traditional electrodynamics, one considers that (because there are many other moving charged particles in the distant universe) there always is the presence of a random electromagnetic background in the form of a random radiation affecting the particle(s) in our experiment. This new boundary condition replaces the null one of traditional classical electrodynamics. Moreover, as the relativity principle dictates that identical experiments performed in different inertial frames must yield the same result, this random classical electromagnetic radiation must be the same in all inertial frames and therefore have a Lorentz-invariant energy density spectrum. But the only energy density spectrum that obeys such a condition happens to be one that is proportional to the cubic power of the frequency. Interestingly enough, this is exactly the same frequency dependence as that of the QED spectral ZPF energy density presented above when in equation (1) we set the temperature $\mathrm{T}$ to zero. In SED we can then write this random radiation in the same way as 
the ZPE of QED and we call it the classical electromagnetic ZPE. Planck's constant appears then in SED as an adjustable parameter that sets the scale of the ZPE spectral density. Several quantum results have been reproduced by means of the classical SED approach. For a very thorough, detailed and scholarly review of SED, see de la Peña and Cetto (1996). This book was reviewed by two of us (Cole and Rueda, 1996). Nevertheless, QED and SED do not in general yield the same results for nonlinear systems, although they are in agreement for the linear systems examined. The apparent disagreements between SED and QED are quite serious, since they occur in areas that QED is highly successful. Speculatively, but quite possibly, the source of these difficulties lies in accurately dealing with the nonlinear stochastic differential equations in SED for these problems. However, even if this can be satisfied, it is most likely there will still be differences that should clearly be testable by experimental means (Cole, 2005).

\section{PROPOSED EXPERIMENTS}

In what follows, we outline each of the proposed experimental concepts that we plan to explore theoretically and in the laboratory, though space limitations and proprietary concerns force us to limit the level of detail that we can present. The experimental and theoretical program described below has undergone preliminary evaluation by Lockheed Martin review panels involving both internal R\&D personnel and outside experts on theory and experimentation.

\section{Voltage Fluctuations in Coils Induced by ZPF at High Frequency}

In a series of experiments, Koch et al. $(1980 ; 1981 ; 1982)$ measured voltage fluctuations in resistive wire circuits that are induced by the ZPF. The Koch et al. result is striking confirmation of the reality of the ZPF and proves that the ZPF can do real work (cause measurable currents). Although the Koch et al. experiment detected miniscule amounts of ZPF energy, it shows the principle of ZPF energy circuit effects to be valid and opens the door to consideration of means to extract useful amounts of energy.

Blanco et al. (2001) have proposed a method for enhancing the ZPF-induced voltage fluctuations in circuits. Treating a coil of wire theoretically as an antenna, they argue that the antenna-like radiation resistance of the coil should be included in the total resistance of the circuit, and they suggest that it is this total resistance that should be used in the theoretical computation of the ZPF-induced voltage fluctuations. Because of the strong dependence of the radiation resistance on the number of coil turns (scaling quadratically), coil radius (quartic scaling), and frequency (quartic scaling), these enhanced ZPF-induced voltage fluctuations should be measurable in the laboratory at quite accessible frequencies (100 MHz compared to the $100 \mathrm{GHz}$ range necessary in the Koch et al. experiments).

The Blanco et al. theory is as follows. Random voltage fluctuations are conveniently described by their frequency spectrum. That is, given a time interval of measured voltages, one can Fourier transform the measurements to the frequency domain to determine how the voltage fluctuations are distributed in frequency (e.g., how much lowfrequency, long duration fluctuations are present relative to high-frequency, short-duration fluctuations). Theoretically the spectrum of voltage fluctuations $\mathrm{S}(\omega, \mathrm{T})$ of a resistive circuit is given by:

$$
S(\omega, T)=\frac{R(\omega, T)}{\pi} \frac{\hbar \omega}{2} \operatorname{coth}\left(\frac{\hbar \omega}{2 k T}\right),
$$

where $\mathrm{R}(\omega, \mathrm{T})$ is the total resistance (ohmic plus radiative), $\omega$ is the (angular) frequency, and $\mathrm{T}$ is the absolute temperature. The resistance $\mathrm{R}(\omega, \mathrm{T})$ is temperature dependent through its ohmic part (the radiation resistance contribution is frequency-dependent only). The postulate of Blanco et al. is that the total resistance must include the radiation resistance of the circuit:

$$
R(\omega, T)=R_{\text {ohmic }}(\omega, T)+R_{\text {rad }}(\omega) .
$$

Under the assumption that the wavelengths of the ZPF modes of interest are larger than the dimensions of the circuit, the radiation resistance of a coil is given by: 


$$
R_{\text {rad }}(\omega)=\frac{2}{3} \frac{\pi^{2} N^{2}}{c}\left(\frac{a \omega}{c}\right)^{4}
$$

where $\mathrm{N}$ is the number of coil turns, and a is the radius of the coil winding.

According to the Blanco et al. theory, large enhancements in ZPF-induced voltage fluctuations are possible. By reducing the temperature to minimize ohmic resistance, making the coil of many turns and large radius, and performing measurements at high frequency, it will be possible to confirm this amplification effect. Using the theory of Blanco et al., the predicted coil-enhanced voltage spectrum can be computed. For a $1 \mathrm{~cm}$ diameter coil of 2000 turns made of 38 AWG Tungsten wire, and at a temperature of $3 \mathrm{~K}$, the result is shown in Figure 4. In Figure 4, the upper (blue) curve represents the predicted voltage spectral density for the combined ohmic plus radiation resistance. The lower (red) curve is the result when radiation resistance is ignored. If the Blanco et al. postulate is correct, the enhancement effect of the coil should be easily measured at frequencies as low as $100 \mathrm{MHz}$ (where the coil enhancement effect is about 100 -fold for Tungsten).

To successfully measure the ZPF-induced voltage fluctuations, the requirements of low temperature, large coil, and high frequency must be met. The low-temperature requirement is met by performing the experiment in a cooled dewar. There are laboratories with high-quality dewars (pumped down to $3 \mathrm{~K}$ ) and sensitive instruments suitable for the measurements. The cold spot in one particular dewar under consideration is cylindrical, $2.5 \mathrm{~cm}$ in diameter and height. Thus the largest coil that we can consider will have a coil radius of approximately a $=1 \mathrm{~cm}$. To keep the linear dimension of the coil small, we will require small wire thicknesses, say $b=0.01 \mathrm{~cm}$ (gauge $38 \mathrm{AWG).} \mathrm{By}$ winding the coil in a number of layers (10 or 12 layers) we can accommodate a large number of turns, say $\mathrm{N}=2,000$ turns. To minimize ohmic resistance, wire made of tungsten $(\mathrm{W})$ is preferred. However, copper $(\mathrm{Cu})$ is a suitable alternative providing significant cost savings.

Voltage fluctuations in the $100 \mathrm{MHz}$ range are easily detected using fairly common laboratory equipment. We could perform this experiment using tungsten without resorting to the sophisticated/costly techniques used by Koch et al. to attain their extremely high frequency measurements (involving resistively shunted Josephson junctions).

For a copper wire coil, the magnitude of the enhancement effect is reduced somewhat compared to the tungsten case of Figure 4, but for frequencies approaching the $\mathrm{GHz}$ regime, the radiation resistance enhancement effect is still over four orders of magnitude. Equipment easily obtained will allow measurements of the voltage spectrum in the $\mathrm{GHz}$ regime. Therefore, given the cost tradeoff of coil fabrication for copper vs. tungsten, we may use copper coils and perform the experiment. Suitable coils will be fabricated by a custom coil-winding vendor, and a second coil will be tested. The second coil will be used in a control experiment. It will be constructed with the same parameters as the first coil, but will have half of its turns wound in the reverse direction. This will make the coil non-inductive so that its voltage spectral density should correspond to the lower red curve in Figure 4.

\section{ZPF Energy Extraction by Ground State Energy Reduction}

As first analyzed by Boyer (1975) and later refined by Puthoff (1987), the following paradox was addressed that even though atomic ground states involve electrons in accelerated motion, such states are nonetheless radiationless in nature. This ground state characteristic was shown to be interpretable (for the standard Bohr ground state orbit of the hydrogen atom) as an equilibrium process in which radiation by the electron in its ground state orbit was compensated by absorption of radiation from the background vacuum electromagnetic ZPE. This interpretation has recently been strengthened by the analyses of Cole and Zou (2003; 2004) using a stochastic electrodynamic (SED) model for the vacuum ZPE. Since the balance between emitted orbital-acceleration radiation and absorbed ZPE radiation is modeled as taking place primarily at the ground state orbital frequency, we can consider the possibility of using this feature in a mechanism to extract energy from the ZPE.

By passing monatomic gas atoms through specially constructed microcavities (e.g., resonant conducting Casimir cavities), which suppress the background vacuum ZPE at the ground state frequency (thereby upsetting the balance), one might expect the ground state orbit to drop in energy to a new equilibrium orbit and release the energy difference (see Figure 5). Such a process would mimic the usual radiation emission of an electron dropping from an 
excited state to the ground state, although in this case the "excited" state is the free-space ground state and the final state is the new lower-energy equilibrium ground state resulting from the effects of cavity mode suppression. Microcavity fabrication to match the atomic ground states is daunting because there will potentially be fabrication irregularities that cause edge and surface effects which act upon the particles as they enter or exit the Casimir region. It will be a challenge to properly account for this so as to disentangle the effects from experimental results. However, further investigation showed that this principle is broadly applicable to other than just atomic ground states, e.g., to the ground state harmonic-oscillator-type vibrational motion of, say, an $\mathrm{H}_{2}$ molecule. The estimated power output under optimized conditions from this experimental approach is estimated to be on the order of Watts to kiloWatts.

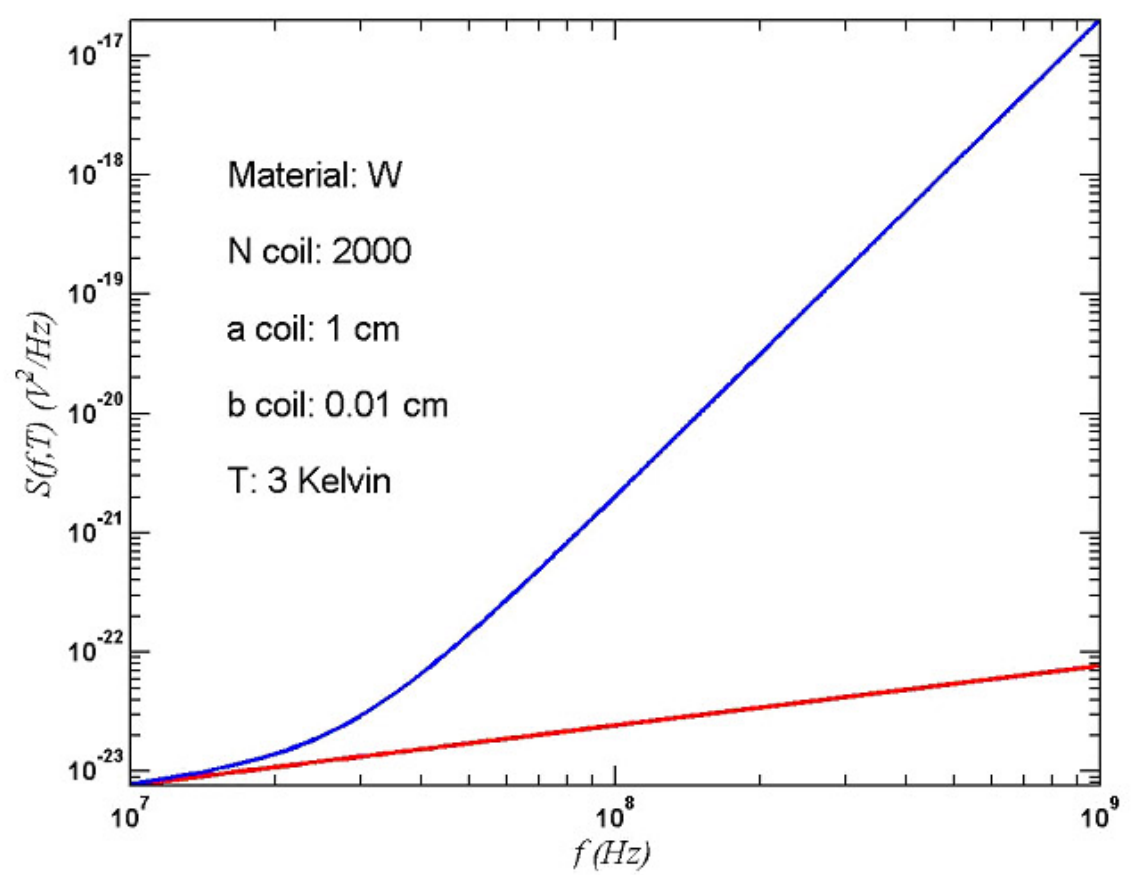

FIGURE 4. Theoretical Voltage Spectral Density of a Tungsten Coil.

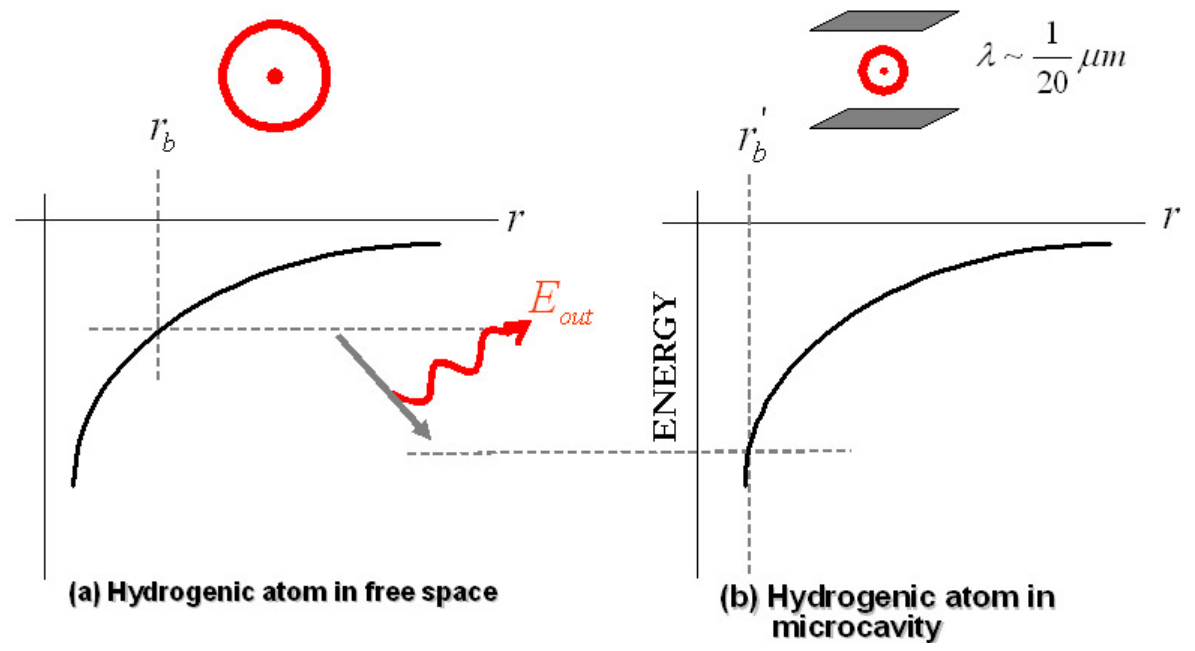

FIGURE 5. Release of Energy ( $\left.\mathrm{E}_{\text {out }}\right)$ from Ground State Suppression of Hydrogenic Atom in a Microcavity. $\left(r_{b}=\right.$ free-space Bohr orbital radius, $r_{b}{ }^{\prime}=$ suppressed Bohr orbital radius, $\lambda=$ resonant wavelength of Bohr orbit $)$ 
An exploratory effort to investigate the above concept was carried out by Puthoff et al. (2001). In their experiment $\mathrm{H}_{2}$ gas was passed through a $1 \mu \mathrm{m}$ Casimir cavity to suppress the ZPE radiation at the vibrational ground state of the $\mathrm{H}_{2}$ molecule (see Figure 6). The anticipated signature for such a process would be increased dissociation energy of the molecule. For this purpose, the experiment was carried out at the Synchrotron Radiation Center at the University of Wisconsin at Madison, where an intense UV beam to disassociate gas molecules could be generated. Unfortunately, problems with the synchrotron beam (unrelated to the experiment) prevented a definitive result from being obtained, so the efficacy of this ZPE-extraction approach remains undetermined at the present time. Further experimentation of this type will be explored with regard to ZPE extraction.
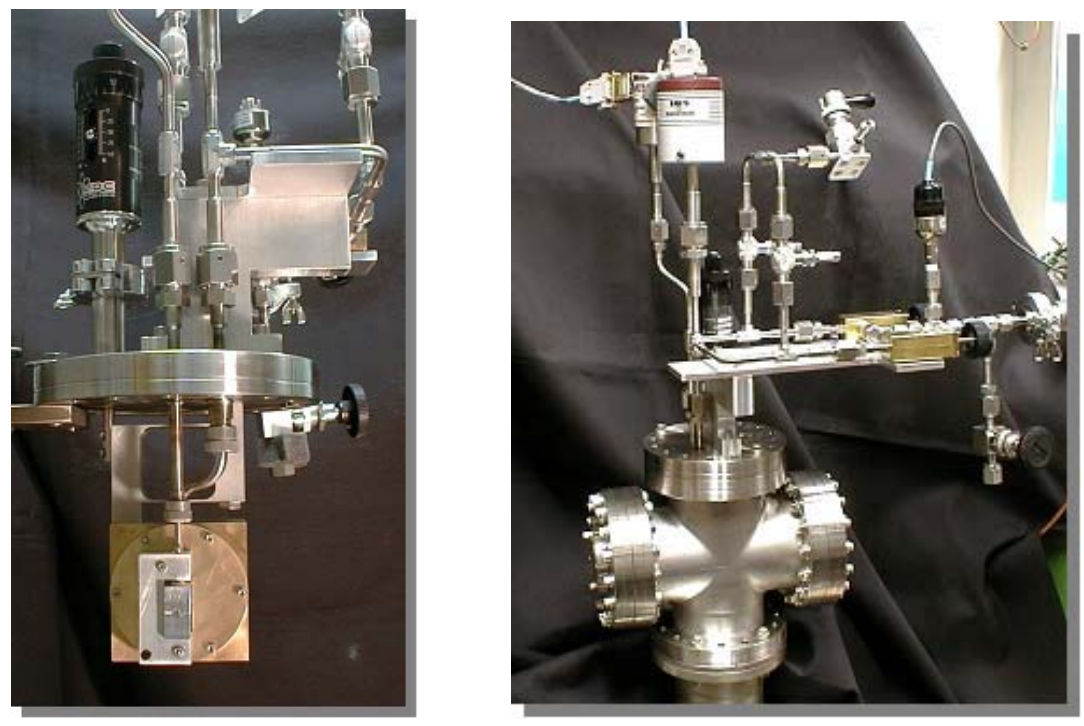

FIGURE 6. Experimental Apparatus for Ground State Energy Reduction Tests.

\section{Tunable Casimir Effect}

The Casimir Effect is a unique ZPF-driven quantum force that occurs between closely-spaced conductive cavity walls (or plates). If left unfettered, the plates will collapse together and energy is converted from the ZPF into heat (or other forms of energy) in accordance with the expression E/A $=\pi^{2} \hbar c / 720 \mathrm{~d}^{3}$, where E/A is the energy per unit area of the plates and $d$ is the plate separation. Further investigation of this mechanism by Cole and Puthoff (1993) showed that this process not only did not violate energy conservation, but it was compatible with thermodynamic constraints as well.

Although the Casimir force is conservative, and thus the Casimir device might appear to be a one-shot device, the fact that the attractive Casimir force is weaker for dielectric plates compared to conductive plates raises the possibility of the use of thin-film switchable mirrors to obtain a recycling engine (Puthoff, 1985; Lipkin, 1996; Pinto, 1999). In such an application the plates are drawn together by the stronger force associated with the conducting state and withdrawn after switching to the dielectric state. Under the most optimistic conditions for eventual embodiment in practical devices (where negligible energy is required for switching, oscillation between 30 $\mathrm{nm}$ and $15 \mathrm{~nm}$ spacing can be achieved for $1 \mathrm{~cm}^{2}$ plates, driving circuit $\approx 10$ times the weight of the Casimir plates, etc.), an estimate based on a switching oscillation from a purely conductive state to a dielectric constant of $\mathrm{K}=4$ yields a figure of merit of $\approx 35 \times \mathrm{f}(\mathrm{MHz}) \mathrm{W} / \mathrm{kg}$ ( $\mathrm{f}=$ switching rate) for the power density (Puthoff, 1985). This can be compared to the power density of $\approx 5 \mathrm{~W} / \mathrm{kg}$ achieved by radioisotope thermoelectric generators. The predicted output power per unit area for this experimental device is $\approx 10^{-6} \mathrm{f}(\mathrm{MHz}) / 4[\mathrm{~d}(\mu \mathrm{m})]^{3}$ Watts $/ \mathrm{cm}^{2}$.

Another "tunable" conductive-type plate experiment under consideration involves the use of plates consisting of three-dimensional photonic crystals with the bandgap of the photons that can transmit through the structure being a "tunable" value. Using microelectromechanical processing methods, Sandia National Laboratories has 
experimentally produced such crystals and are researching methods of actively modifying the structures while in use (Lin et al., 2003). Finally, the technology requirements for this experimental concept are the nano-fabrication of microcavities with thin-film deposited surfaces, RF-driven piezoelectric mounts for cavity oscillation, mirrorswitching modality (e.g., hydrogen pressure modulation), and calorimetric measurement of energy/heat production.

\section{Electron Inertial Mass Test}

Another interesting concept to explore is a postulated electron mass change caused by the exclusion of ZPF modes between Casimir plates, which would result in a change in the optical path-length of a laser beam passing through the cavity compared to a beam not passing through it. According to the quantum vacuum inertia hypothesis (Haisch et al., 1994; 2001; Rueda and Haisch, 1998a; 1998b; 2005), as well as the connectivity approach of Nickisch and Mollere (2002), at least some component of inertial mass derives from charge interactions with the ZPF. It is possible to investigate the electromagnetic basis of inertial mass experimentally by using Casimir plates immersed in a plasma. The Casimir plates are enclosed in a vacuum tube in which uniformly distributed plasma is created. A laser beam is split three ways, as shown in Figure 7. One laser beam is directed between the Casimir plates, and the other two outside the plates. The goal is to combine Beams 1 and 2, and, separately, Beams 2 and 3 and to look for interference fringe shifting of the 2-3 combination relative to the 1-2 combination. Under the postulated effect this fringe shift would be due to an inertial mass change of the electrons between the plates. Note that the electron plasma frequency $\mathrm{f}_{\text {plasma }}$ is given (in Gaussian units) by:

$$
f_{\text {plasma }}=\sqrt{\frac{4 \pi N_{e} e^{2}}{m_{e}}},
$$

where $\mathrm{N}_{\mathrm{e}}$ is the electron number density, e is the electron charge, and $\mathrm{m}_{\mathrm{e}}$ is the electron mass. The index of refraction of the plasma is given by:

$$
n=\sqrt{1-\frac{f_{\text {plasma }}^{2}}{f^{2}}}
$$

where $f$ is the frequency of the laser. Any electron mass change caused by the exclusion of ZPF modes between the Casimir plates would result in a change in the optical path-length for Beam 3 and produce a corresponding interference fringe shift. It might be possible to attach the plates to a device for changing the plate separation and look for interference fringe shifting as a function of plate separation. Alternatively, a Casimir tube could be used instead of plates for enhanced effect since the Casimir ZPF exclusion would then occur in two dimensions instead of only one. We will be investigating the feasibility of this experiment, including working out the best combination of laser frequency and plasma density and calculating the magnitude of the effect expected.

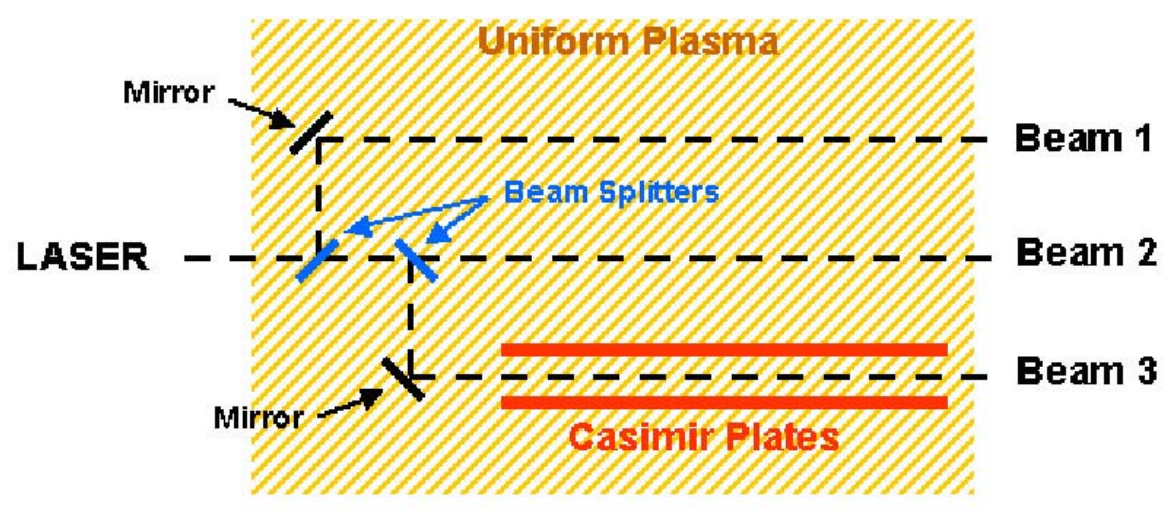

FIGURE 7. Electron Inertial Mass Test. 


\section{CAVEAT EMPTOR!}

There has been a large amount of popular semi-technical literature published over the last 25 years covering the topic of extracting energy from the quantum vacuum field. The literature is mostly composed of self-published books or pamphlets found in bookstores or on the internet, and there are also professional society conference papers that are largely not peer-reviewed. Unfortunately, much of this literature is published within the context of free energy and antigravity devices with claims that the vacuum ZPE is the source that drives free energy devices or powers an antigravity craft, or powers gravity/mass modification or repulsive gravitational force beam devices, etc. A number of these claims have been evaluated over the years by credentialed scientists and were falsified. Much of this literature is self-serving marketing propaganda, and the language describing the physics or engineering principles for these claims is often couched in what we call "technobabble." Credentialed scientists interested in seriously pursuing a laboratory investigation of the vacuum ZPF should be forewarned that many of the claims being made in the non-peer-reviewed literature are fraught with pathological science, fraud, misinformation, disinformation, and spurious physics. This is the reason why the present authors were very selective about which ZPE extraction approaches to consider for our research program.

\section{CONCLUSION}

We reviewed the physical nature of the quantum vacuum field, and described its spectral characteristics and latent energy content. We are interested in concepts that provide an experimental framework for exploring the possibility and limitations of accessing energy from the space vacuum environment. The theoretical approaches guiding this experimental investigation are based on the QED and SED models of the ZPF. The purpose of our investigation is to explore the question of whether the quantum vacuum field contains useful energy that can be exploited for space power and propulsion applications under the action of a catalyst, or cavity structure, so that energy conservation is not violated. We identified six experiments that have the potential to extract useful energy from the vacuum. One of these, Forward's Vacuum-Fluctuation Battery, was shown to be unsuitable for completing an engine cycle for pumping energy from the vacuum. The efficacy of the Mead and Nachamkin patent device has not yet been evaluated in the lab. However, four additional experimental concepts are potentially exploitable and we have selected those to pursue in a carefully guided theoretical and laboratory research program. The estimated power output from three of these concepts could under optimum conditions range from Watts to kiloWatts. But it should be stressed that there potentially is a real theoretical and experimental challenge in modeling and predicting noise sources, edge and surface effects, etc. within the different experimental approaches, so that experimental results are unambiguously interpretable. If successful, however, it is anticipated that these experiments would lead to a revolution in the way we generate electrical power for commercial and space applications.

\section{ACKNOWLEDGEMENTS}

The authors wish to thank Lockheed Martin Space Systems, the Institute for Advanced Studies at Austin, ManyOne Networks, Boston University, and California State University-Long Beach for their institutional support of this research program. We are also grateful to Jeff Newmeyer (Lockheed Martin) for very useful comments.

\section{REFERENCES}

Blanco, R., França, H. M., Santos, E., and Sponchiado, R. C., "Radiative noise in circuits with inductance,” Phys. Lett. A 282, 349-356 (2001).

Boyer, T. H., "Random electrodynamics: The theory of classical electrodynamics with classical electromagnetic zero-point radiation," Phys. Rev. D 11, 790-808 (1975).

Casimir, H. B. G., "On the Attraction Between Two Perfectly Conducting Plates," Proc. Kon. Ned. Akad. Wetensch. 51, 793-796 (1948).

Chen, F., et al., "Theory confronts experiment in the Casimir force measurements: Quantification of errors and precision," Phys. Rev. A 69, 022117 (2004).

Cole, D. C., "Simulation Results Related to Stochastic Electrodynamics," in proc. of the int'l conf. Quantum Theory:

Reconsideration of Foundations-3, AIP Press, 2005, in press. 
Cole, D. C., and Puthoff, H. E., "Extracting energy and heat from the vacuum," Phys. Rev. E 48, 1562-1565 (1993).

Cole, D. C., and Rueda, A., "The Quantum Dice: An Introduction to Stochastic Electrodynamics," Found. Phys. 26, 1559-1562 (1996).

Cole, D. C., and Zou, Y., "Quantum Mechanical Ground State of Hydrogen Obtained from Classical Electrodynamics," Phys. Lett. A 317, 14-20 (2003).

Cole, D. C., and Zou, Y., "Analysis of Orbital Decay Time for the Classical Hydrogen Atom Interacting with Circularly Polarized Electromagnetic Radiation," Phys. Rev. E 69, 016601 (2004).

de la Peña, L., and Cetto, A. M., The Quantum Dice: An Introduction to Stochastic Electrodynamics, Kluwer, Dordrecht, Holland, 1996.

Forward, R. L., Alternate Propulsion Energy Sources, Final Report AFRPL TR-83-067, Air Force Rocket Propulsion Laboratory, Air Force Space Tech. Ctr. Space Div., Air Force Systems Command, Edwards AFB, CA, 1983, pp. A1-A14.

Forward, R. L., "Extracting Electrical Energy from the Vacuum by Cohesion of Charged Foliated Conductors," Phys. Rev. B 30, 1700-1702 (1984).

Haisch, B., Rueda, A., and Dobyns, Y., "Inertial mass and the quantum vacuum fields," Ann. der Phys. 10, 393-414 (2001).

Haisch, B., Rueda, A., and Puthoff, H. E., "Inertia as a zero-point-field Lorentz force," Phys. Rev. A 49, 678-694 (1994).

Koch, R. H., Van Harlingen, D. J., and Clarke, J., "Quantum-Noise Theory for the Resistively Shunted Josephson Junction," Phys. Rev. Lett. 45, 2132-2135 (1980).

Koch, R. H., Van Harlingen, D. J., and Clarke, J., "Observation of Zero-Point Fluctuations in a Resistively Shunted Josephson Tunnel Junction," Phys. Rev. Lett. 47, 1216-1219 (1981).

Koch, R. H., Van Harlingen, D. J., and Clarke, J., "Measurements of quantum noise in resistively shunted Josephson junctions," Phys. Rev. B 26, 74-87 (1982).

Lamoreaux, S. K., "Demonstration of the Casimir Force in the 0.6 to $6 \mu \mathrm{m}$ Range," Phys. Rev. Lett. 78, 5-8 (1997).

Lin, S. Y., Moreno, J., and Fleming, J. G., "Three-dimensional photonic-crystal emitter for thermal photovoltaic power generation," Appl. Phys. Lett. 83, 380-382 (2003).

Lipkin, R., "Thin-film mirror changes into a window," Science News 149, 182 (1996).

Mead, F. B., and Nachamkin, J., "System for Converting Electromagnetic Radiation Energy to Electrical Energy," United States Patent No. 5,590,031 (1996)

Milonni, P. W., The Quantum Vacuum: An Introduction to Quantum Electrodynamics, Academic Press, New York, 1994, pp. 133, 290-294.

Mohideen, U., "Precision Measurement of the Casimir Force from 0.1 to $0.9 \mu \mathrm{m}$," Phys. Rev. Lett. 81, 4549-4552 (1998).

Nickisch, L. J., and Mollere, J., “Connectivity and the Origin of Inertia,” arxiv.org/PS_cache/physics/pdf/0205/0205086.pdf (2002).

Peres, A., Quantum Theory: Concepts and Methods, Kluwer, Dordrecht, Holland, 1993.

Pinto, F., "Engine cycle of an optically controlled vacuum energy transducer," Phys. Rev. B 60, 14740-14755 (1999).

Puthoff, H. E., "Vacuum energy extraction by conductivity switching of Casimir force," Technical Memo, Inst. for Advanced Studies at Austin, 1985.

Puthoff, H. E., "Ground state of hydrogen as a zero-point-fluctuation-determined state," Phys. Rev. D 35, 3266-3269 (1987).

Puthoff, H. E., Little, S. R., and Ibison, M., "Discussion of experiment involving lowering of ground state energy for oscillation in a Casimir cavity," Int'l Conf. on Squeezed States and Uncertainty Relations (ICSSUR 2001): Special Session on Topics of Physics Related to Stochastic Electrodynamics, Boston University, 2001.

Rueda, A., and Haisch, B., "Inertia as reaction of the vacuum to accelerated motion," Phys. Lett. A 240, 115-126 (1998a).

Rueda, A., and Haisch, B., "Contribution to inertial mass by reaction of the vacuum to accelerated motion," Found. Phys. 28, 1057-1108 (1998b).

Rueda, A., and Haisch, B., “Gravity and the quantum vacuum inertia hypothesis,” Ann. der Phys. 14, 479-498 (2005). 